\title{
Hämatopoetische Stammzelltransplantation
}




\section{Hämatopoetische Stammzell- transplantation}

Neue Konzepte in der Rehabilitation und Nachsorge transplantierter Patienten

Herausgeber

H.H. Bartsch, Freiburg i.Br.

J. Finke, Freiburg i.Br.

A. Mumm, Freiburg i.Br.

39 Abbildungen, 14 farbig, 29 Tabellen, 2001

\section{Tumortherapie und Rehabilitation}

Freiburger Beiträge herausgegeben von H.H. Bartsch 
Deutsche Bibliothek - CIP-Einheitsaufnahme

Hämatopoetische Stammzelltransplantation: neue Konzepte in der Rehabilitation und Nachsorge transplantierter Patienten; 29 Tabellen / Hrsg. H.H. Bartsch . . - Basel; Freiburg [Breisgau]; Paris; London; New York; New Delhi; Bangkok; Singapore; Tokyo; Sydney: Karger, 2001

(Tumortherapie und Rehabilitation)

ISBN 3-8055-7234-4

0101 deutsche buecherei 0292 deutsche bibliothek

Dosierungsangaben von Medikamenten

Autoren und Herausgeber haben alle Anstrengungen unternommen, um sicherzustellen, dass die Auswahl und Dosierungsangaben von Medikamenten im vorliegenden Text mit den aktuellen Vorschriften und der Praxis übereinstimmen. Trotzdem muss der Leser im Hinblick auf den Stand der Forschung, Änderung staatlicher Gesetzgebungen und den ununterbrochenen Strom neuer Forschungsergebnisse bezüglich Wirkung und Nebenwirkungen von Medikamenten darauf aufmerksam gemacht werden, dass unbedingt bei jedem Medikament die Packungsbeilage konsultiert werden muss, um mögliche Änderungen im Hinblick auf die Indikation und Dosierung nicht zu übersehen. Gleiches gilt für spezielle Warnungen und Vorsichtsmaßnahmen. Ganz besonders gilt dieser Hinweis für empfohlene neue und/oder nur selten gebrauchte Wirkstoffe.

Alle Rechte vorbehalten. Ohne schriftliche Genehmigung des Verlags dürfen diese Publikation oder Teile daraus nicht in andere Sprachen übersetzt oder in irgendeiner anderen Form mit mechanischen oder elektronischen Mitteln (einschließlich Fotokopie, Tonaufnahme und Mikrokopie) reproduziert oder auf einem Datenträger oder einem Computersystem gespeichert werden.

(C) Copyright 2001 by S. Karger GmbH, Postfach D-79095 Freiburg, und S. Karger AG, Postfach, CH-4009 Basel

Printed in Germany on acid-free paper by Druckhaus «Thomas Müntzer» GmbH,

Bad Langensalza

ISBN 3-8055-7234-4 


\section{Inhalt}

Vorwort der Herausgeber. . . . . . . . . . . . . . VII

Stammzelltransplantation 2000 - Quo vadis? . . . . . . . . . . . 1

Gratwohl, A. (Basel)

Rehabilitation nach hämatopoetischer Stammzelltransplantation . . . . . 10

Mumm, A.; Willenbacher, W.; Bartsch, H.H. (Freiburg i.Br.)

Freiburger Standards der ambulanten Nachsorge nach allogener häma-

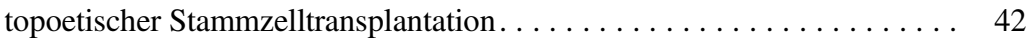

Bertz, H. (Freiburg i.Br.)

Die rehabilitative Arbeit mit Patienten nach hämatopoetischer Stammzelltransplantation aus psychologischer Sicht $\ldots \ldots \ldots \ldots \ldots \ldots \ldots \ldots \ldots$

Birmele, M. (Freiburg i.Br.)

Therapierelevante Parameter der immunologischen Rekonstitution nach allogener hämatopoetischer Stammzelltransplantation . . . . . . . . 58

Einsele, H.; Hebart, H. (Tübingen)

«Graft versus host»-Reaktion - Stellenwert neuer Immunsuppressiva . . . .

Kiehl, M.G.; Fauser, A.A. (Idar-Oberstein)

Ophthalmologische Probleme nach allogener hämatopoetischer Stamm-

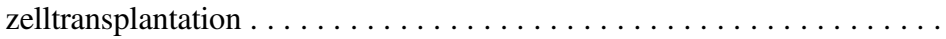

Mittelviefhaus, H. (Freiburg i.Br.)

Pneumologische Probleme nach allogener Stammzelltransplantation. . . . .

Holler, E.; Pfeifer, M.; Hahn, J.; Hildebrandt, G.; Gerbitz, A.; Reichle, A.; Müller, T. (Regensburg); Kolb, H.J. (München); Andreesen, R.

(Regensburg) 
Endokrine Probleme nach allogener hämatologischer Stammzelltrans-

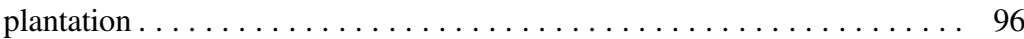

Reincke, M; Lutz, A.; Slawik, M.; Beuschlein, F. (Freiburg i.Br.)

Die Ernährung in der Intermediär- und Spätphase nach allogener hämatopoetischer Stammzelltransplantation . . . . . . . . . . . . . . . . . . . 105

Zürcher, G. (Freiburg i.Br.)

Pflegerische Aufgaben im Rahmen der Rehabilitation nach hämatopoetischer Stammzelltransplantation $\ldots \ldots \ldots \ldots \ldots \ldots \ldots \ldots \ldots \ldots \ldots$

Thieme, G. (Freiburg i.Br.)

Hygieneanforderungen im Rahmen der Rehabilitation nach hämatopoeti-

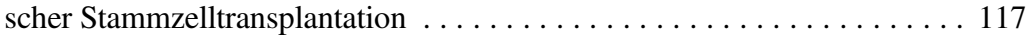

Salrein-Hahn, G. (Freiburg i.Br.)

Physio- und Sporttherapie nach hämatopoetischer Stammzelltransplantation.

Hochuli, P.; Boltz, B.; Mumm, A. (Freiburg i.Br.)

Lebensqualität und psychosoziale Integration bei stammzell- oder knochenmarktransplantierten Patienten nach stationärer Rehabilitation

Weis, J; Mumm, A.; Willenbacher, W.; Ehlers, K.; Bartsch, H.H.

(Freiburg i.Br.)

Neuropsychologische Diagnostik und Therapie nach Hochdosis-Therapien

Poppelreuter, M. (Freiburg i.Br.)

Integrative psychosoziale Unterstützung im Rahmen stationärer und ambulanter Nachsorge nach hämatologischer Stammzelltransplantation . . . . . . 163

Schulz-Kindermann, F. (Hamburg)

Sozialrechtliche Fragen nach hämatologischer Stammzelltransplantation . . 171 Fild, H.C. (Freiburg i.Br.)

Das Fatigue-Syndrom in der Onkologie . . . . . . . . . . . . . . . . 178

Flechtner, H. (Köln) 


\section{Vorwort der Herausgeber}

In der Reihe «Tumortherapie und Rehabilitation» ist dies bereits der zweite Band, der sich mit Problemen und Folgestörungen nach Stammzelltransplantation befasst. Die Transplantation von Knochenmark und peripheren hämatopoetischen Stammzellen hat sich in den letzten 5 Jahren seit dem Erscheinen des ersten Bandes rasant weiter entwickelt. In qualitativer Hinsicht spiegelt sich diese Weiterentwicklung einerseits in einer Verbesserung der Heilungschancen der behandelten Patienten und andererseits in einer Verringerung der akuten, mittel- und langfristigen Komplikationen und Folgestörungen nach Transplantation wider. Gleichzeitig sind, verglichen mit den Jahren zuvor, deutlich mehr Transplantationen von Stammzellen durchgeführt worden. So stieg die Anzahl der in Deutschland pro Jahr durchgeführten hämatopoetischen Stammzelltransplantationen allein von 1995 bis 1998 von zirka 1600 auf 3500. Hierdurch sind alle Berufsgruppen, die sich an der Nachbetreuung dieser Patienten beteiligen, besonders gefordert.

Der vorliegende Buchband gibt einen aktuellen Überblick zu diesem Thema und beleuchtet wesentliche Aspekte im Zusammenhang mit hämatologischen Transplantationen aus der Sicht der verschiedenen an der Betreuung der transplantierten Patienten beteiligten Fachdisziplinen. Die einzelnen Beiträge gehen auf das interdisziplinäre Symposium «Ambulante Nachsorge und stationäre Rehabilitation nach allogener hämatopoetischer Stammzelltransplantation» zurück, das vom 13. bis 14. April 2000 im Tumorzentrum Freiburg stattfand. Das breite Spektrum der behandelten Themen spiegelt die Komplexität der Probleme und relevanten Fragen wider. Hierzu gehören die Strategien der ambulanten Nachsorge und der stationären Rehabilitation ebenso wie das aktuelle Wissen über die posttransplantäre hämatopoetische und immunologische Rekonstitution. Breiten Raum findet die Darstellung mittel- und langfristiger Folgen und deren Therapie sowie Probleme der Pflege, Hygiene, Sozialmedizin, Psychoonkologie und der Neuropsychologie.

Der einführende Beitrag von Gratwohl fasst den derzeitigen Stand der Stammzelltransplantation zusammen und gibt einen Ausblick auf mögliche Entwicklungen dieses therapeutischen Verfahrens. Bertz beleuchtet aktuelle 
Standards der ambulanten Nachsorge nach Stammzelltransplantation. Die Beiträge von Mumm et al. und Birmele beschreiben, basierend auf ihren Erfahrungen an der Klinik für Tumorbiologie Freiburg i.Br., zusammenfassend die vielfältigen Probleme der Rehabilitation von Patienten nach Stammzelltransplantation. Möglichkeiten der Diagnostik der immunologischen Rekonstitution nach allogener hämatopoetischer Stammzelltransplantation werden von Einsele und Hebart dargestellt. Der Beitrag von Kiehl und Fauser gibt einen Überblick zum Stellenwert neuer Immunsuppressiva in der Behandlung der «Graft versus host»-Reaktion, die eines der gravierendsten Probleme nach einer Stammzelltransplantation darstellt. Mittelviefhaus, Holler et al. und Reincke et al. beschreiben Folgestörungen der hämatopoetischen Stammzelltransplantation aus ophthalmologischer, pneumologischer und endokriner Sicht. Aufgrund der posttransplantären Immunsuppression der transplantierten Patienten kommt der Hygiene und der Ernährung in der Nachsorge eine besondere Bedeutung zu. Zürcher geht in ihrem Beitrag auf die unterschiedlichen Ernährungsbedürfnisse im Verlauf der Rehabilitation nach Stammzelltransplantation ein, während SalreinHahn die Hygieneanforderungen in der Nachsorge dieser Patienten beleuchtet und Thieme auf pflegerische Aufgaben eingeht. Dass neben der medikamentösen Behandlung auch die Aktivierung durch Sporttherapie ein wichtiger Behandlungsansatz in der Rehabilitation von stammzelltransplantierten Patienten ist, zeigen Hochuli et al. Mit zunehmender Verbreitung der hämatopoetischen Stammzelltransplantation setzte auch eine intensive Beschäftigung mit psychosozialen Aspekten, wie Lebensqualität und psychosoziale Integration, nach erfolgreicher Transplantation ein. So geht Poppelreuter auf neuropsychologische Folgen der Transplantation und deren Therapie ein. Die Beiträge von Weis et al. und von Schulz-Kindermann beschäftigen sich hingegen mit Problemen der Lebensqualität und der psychosozialen Reintegration, sowie Konzepten psychosozialer Unterstützung im Rahmen der stationären und ambulanten Nachsorge. Sozialrechtliche Aspekte wie Kostenübernahme, Krankengeld, Rentenzahlung, die für den Patienten oft ein schwer durchschaubares, ihn aber existentiell betreffendes Geflecht bilden, werden von Fild analysiert. Abschließend geht Flechtner auf das Fatigue-Syndrom ein, von dem viele Patienten nach Stammzelltransplantation betroffen sind und das ein schwerwiegendes Problem für die Lebensqualität und psychosoziale Reintegration dieser Patienten darstellt.

Das vorliegende Buch ist ein aktuelles Kompendium zu zahlreichen Therapiefolgestörungen nach hämatopoetischer Stammzelltransplantation. Allen in die Nachsorge eingebundenen Berufsgruppen soll es Orientierungshilfe bei der täglichen Arbeit sein und Anregungen geben. Denjenigen, die sich neu mit der Materie auseinandersetzen, soll es den Einstieg in die vielfältigen mit der hämatopoetischen Stammzelltransplantation verbundenen, somatischen und psychosozialen Problemfelder erleichtern. Wir hoffen, dass dieses Buch eine ebenso große Resonanz wie unser Berichtsband aus dem Jahre 1996 erhält.

In den letzten 5 Jahren haben sich Patienten-Selbsthilfegruppen und Eigeninitiativen Leukämie- und Lymphomkranker unter dem Dach der Deutschen 
Leukämiehilfe mit Sitz in Bonn zusammengeschlossen (DLH, www.leukämiehilfe.de). Auch dies ist eine erfreuliche Entwicklung.

Unser besonderer Dank gilt allen beteiligten Autoren dafür, dass sie es neben ihrer täglichen klinischen Arbeit ermöglicht haben, die Beiträge zu diesem Buch zu verfassen.

Hans Helge Bartsch, Jürgen Finke, Andreas Mumm 